\title{
Inclusive Education in Armenia
}

\author{
Syuzanna Tadevosyan \\ Russian-Armenian (Slavonic) University
}

\begin{abstract}
The article is on the Inclusive Education in general and the status of it in the Republic of Armenia. It mainly covers the notion of Inclusive Education, the functioning model of education in Armenia, its necessity and importance to be applied in many schools of Armenia. The subject is not new but still, there is a lack of information and awareness of it among the people living in Armenia, particularly parents and teachers. Some strategies and suggestions on the topic are included in the article too, that is how to develop and implement it in everyday schooling. Much attention is paid to the training of teachers and the connection of multidisciplinary bonds. Research in a few schools of Armenia proves that the issue is to be well observed to find proper solutions and to provide relevant information and implication of special education at schools and organizations willing to support Inclusive Education.
\end{abstract}

Key words: inclusive education, disability, special needs, equal opportunities, qualification, research, integrate.

\section{Introduction}

Inclusive Education is not a new idea. Communities practiced inclusion throughout centuries. Through the years there was a "common misunderstanding of inclusion related to (incorrectly) considering integration and inclusion to be synonyms; viewing inclusion as simply the presence of a child who is labeled 'disabled' or 'different' in a mainstream setting; thinking that inclusion is only about some people (instead of about everyone); and viewing inclusion as a process of assimilation" (Stubbs 2008:19). 
Much research has been done in this field the majority of which shows that learning in the same school with children without any disability significantly helps the children with disabilities. In the past children with disabilities attended separate schools, but today they are in the same school and sit next to those without any disabilities. This is rather humane and gives grounds not to separate someone from the society. That kind of approach is very valued as it teaches students to support each other realizing that everyone has equal opportunities in the society. Inclusive Education is about building friendship and relationship with all in your group and surrounding. In many countries children with special needs attend special schools and are still separated from the society. Armenia is not an exception in this aspect. Children with special needs are discriminated in everyday activities in schools. We face a real problem today. "All children have a right to survive, thrive and fulfill their potential - to the benefit of a better world" (World Health Organization, "World Report on Disability", 2011:4).

People should learn that Inclusive Education is not a privilege; it is the right of each child.

\section{Representation of Disabilities in Societies}

Thus, why Inclusive Education is important, how Inclusive Education works and what kind of organizations support this education?

As has been mentioned, Inclusive Education is not a privilege, it is just everyone's fundamental right to access education and not be excluded. Yet, there are groups of people who do not have access to this right.

Children with special needs are also a natural part of our world. Their way of learning differs from ours. Methods of Inclusive Education help us work together, help each other and be a part of this world. It is a procedure for expanding support and lessening rejection in a way that adequately reacts to the differing needs of all learners. It includes the individual educating and adapting needs of all minimized and powerless people, including road kids, young ladies, kids from ethnic minorities, kids from financially burdened families, kids from migrant/exile/uprooted families, kids with HIV/AIDS and handicap kids. This 
type of education gives a guarantee to have a good education and not be pushed aside from our society.

Education is a privilege and is a method for acknowledging different rights. It is important to understand the common, political and financial privileges of all kids and young people. It means to battle the minimization of people and to advance contrast. Inclusive Education gains more and more support day by day. The concept of it, nowadays, is wildly spread all over the world.

There are many forms of education based on particular philosophies, spiritual and religious belief systems. But the system is always based on local culture and people.

"Everyone has the right to education. Education shall be free, at least in the elementary and fundamental stages. Elementary education will be compulsory." (The Universal Declaration of Human Rights 1948, Article 26).

\section{Inclusive Education in Armenia}

Education in Armenia has always been highly rated. Armenians have always attached great importance to education, and today the development of the education system and its compatibility with international standards are considered to be among the most important national issues.

In the early 2000s, Inclusive Education was announced as a priority by the Armenian Government. The challenge of developing an inclusive approach to education has been put on the political agenda not only in Armenia but also in many countries around the world. But as the idea of special education is new, not all the Armenians understand it in the right way. It is something new for us. The idea of Inclusive Education becomes popular day by day in Armenia, and we will be discussing its progress here, but still not all people living in Armenia have a clear understanding of it.

In every country the system of Inclusive Education and the way people perceive it differs from a cultural point of view. Being a part of the Armenian society and knowing its culture well enough, I have an opportunity to view the Inclusive Education from the Armenian stand-point. I have had an exclusive possibility to explore the negative and positive sides of Inclusive Education in 
the Republic of Armenia. In addition to my own studies in the field, I found information from different websites, which helped me understand how Armenian culture affects the system of education on children with special educational needs. Thus, my article is precisely on the formation of Inclusive Education in the Republic of Armenia and its peculiarities. I have also tried to make some suggestions.

It has been for about more than 15 years that Inclusive Education has been in existence in the Republic of Armenia. Nevertheless, the lack of information among the parents gives rise to understandable concerns about the impact of inclusion on themselves and their children. They may be unaware of the benefits of inclusion that is offered to all children in the society. They may believe that inclusion is a means of saving money by "dumping" children with disabilities in regular classrooms or recreational programs. Parents who do not have children with special educational needs may think that inclusion will negatively influence the time and attention available for their own children. Some parents of the students without any disabilities believe that Inclusive Education is not effective and that children with disabilities hinder their children's education. At the same time, parents of the children with disabilities have little information about inclusion and special education. Not only disinformation becomes a source of a problem but also the lack of money in some families who do not have information about special education and organizations supporting it.

The Armenian Government adopted the concept of Inclusive Education in Armenia in 2005. In June 2005, the Law on the Education of Persons with Special Education Needs was passed and Inclusive Education was included in the draft of Education Development National Program for 2008-2015. However, the transition from the dual system inherited from Soviet times towards Inclusive Education has not been so far fully implemented. Children with disabilities are most often educated in separate environments, such as special schools. When we look closer at the monitoring initiatives, we can see or highlight the weaknesses in the current educational provisions. Some official 
statistics demonstrates a significant decline in the number of children enrolled in special schools.

However, there has been a huge progress in Inclusive Education during the last decade and that is due to the efforts of professionals.

As a result of my survey, the parents of the children with Special Education Needs (SEN) mentioned the insufficient conditions and low quality of education as the main reasons of exclusion of their children from the mainstream schools. Somehow, we can agree with these parents, because, in many schools located in the villages of Armenia the staff is not appropriately qualified. In other words, there is a problem concerning the lack of education among teachers.

Today the inclusion of children with SEN is a big challenge for many schools, especially in villages and communities. There are still many children with SEN of whom local administrations, schools, and Disabled People's Organizations (DPOs) are unaware. My observations proved that in local schools some parents do not really understand the importance of education for their children and they believe that it is unnecessary, since they feel that $\mathrm{s} / \mathrm{he}$ will never be able to work or get married. During my studies I have not found information about trainings for teachers (not regular). I think that it would be useful if there were trainings for parents too. The trained parents will look at the problem the other way round. If they were educated and had clear information about their child's disability or Inclusive Education, there would not be any conflict between teachers and parents. This is because of absence of any information on this aspect.

In Armenia children with SEN need something more than Inclusive Education. On the basis of my observations in very few Yerevan basic schools, I would conclude that Inclusive Education is not always a good idea (for many parents). Not all the children with SEN should go to school and learn all the things that others without SEN do. It might be more effective if some children with mental disabilities learn some handicrafts instead of going to public schools. In Armenia instead of Inclusive Education, there is a functioning model of integrated education. Children with SEN are just integrated into schools and get the same education as everybody else. But here comes another problem. 
These children are labeled "inclusive kids". So we can come up with the idea that the concept of the quality of Inclusive Education is not present in any form in the Inclusive Education System of Armenia. To improve the situation, the current model of Inclusive Education should be revised. Children with SEN must not just join the classes where children without SEN study. They cannot do all the things that other children do. The quality of education of children with SEN must be guaranteed.

There is another thing which has a negative impact on the quality of Inclusive Education in Armenia. It is the lack of comprehensive approach towards the professional team. In villages and some communities, teachers do not realize the importance of trainings. They do not deeply understand what inclusion is and who the children with SEN are. They do not know how to work with them. The members of multidisciplinary teams do not have a clear understanding of their responsibilities. Subject teachers are indifferent to Inclusive Education and do not devote enough time to educating children with SEN. Sometimes it is not their fault. Not all schools are financed well while the increase of the funding could somehow solve this problem.

State regulations on the structure and functions of multidisciplinary teams should be elaborated too. The team should, at minimum, include a special educator, a social worker, a psychologist and a speech therapist. The structure and functions of the multidisciplinary team must be embedded in a certain Charter and be applicable to all inclusive schools. That is why it is important to decide on the most optimal and child-centered timeframe for special education needs assessments.

We also can highlight another problem connected with the collaboration between multidisciplinary teams, subject teachers and parents of children with SEN. So far, their collaboration has been very weak. Subject teachers do not trust multidisciplinary team members, and parents do not participate in many events connected with their children. There are biased and stereotypical social attitudes towards children with SEN, which are often reinforced by the parents of the children with SEN. Not only parents but also teachers and other students should support each other and learn to work together. More than that, not only 
teachers should have trainings but also parents of the children with SEN should have trainings on how to work with the child or on how important the role of multidisciplinary teams is. Otherwise, misunderstanding and lack of knowledge will become the cause of conflict between teachers and parents.

\section{Key Ingredients of Successful and Sustainable Inclusive Education}

Having identified the common understanding of the phenomenon of Inclusive Education and the people involved in it, several people who were trained in some organizations and were interested in Inclusive Education suggested carrying on some improvements based on their learning experience and attitudes. They elaborated some features to be applied in order to raise awareness among teachers and parents dealing with Inclusive Education. What is relevant to do is to:

1. Prepare training profiles: As multidisciplinary teams consist of various specialists, each member needs to acquire new knowledge and skills relevant to their specific professional functions and needs.

2. Increase access to supplementary training: The members of regional multidisciplinary teams have to commute to the capital for supplementary training, which is quite time-consuming. Creating regional resource centers would partly solve the problem.

3. Increase availability of Inclusive Education materials: The IE materials provided at trainings must be updated. Many teachers do not know where to go to acquire up-to-date literature on IE, since no thematic materials are available in the training section of the NIE (National Institute of Education) website.

4. Increase frequency of trainings: The frequency of training courses is very limited and ineffective in terms of strengthening and enhancing the applicability of knowledge.

5. Provide needs assessment: The exact form of treatment depends on the child's individual needs, and includes understanding of the child's strengths and deficits, as well as what motivates the child, and what is the child's best learning modality. In most cases, a combination of treatment methods is more effective. 
My observations, the experience of teachers and the available information allow us to formulate some teaching tips which can be useful for the ones who want to have success in teaching children with autism. According to Sue Stubbs (2008:5), "Teaching tips are divided into three main groups: communication, social interaction, behavior, each of which has its subgroups".

\section{Communication}

- Provide order and structure to help the person feel less anxious and more comfortable.

- Make a class schedule out of pictures or drawings (for example, song, prayer, story or lesson activity). Point to each picture as you progress through the schedule.

- Be flexible; your schedule must be able to be adapted to the needs of the person with autism.

- Keep instruction short and simple to get a child's attention who has autism, get down on the same level to speak to him or her.

\section{Social Interaction}

- Learn what the person does well (such as putting together puzzles or singing), and find ways to help the person use those skills in the classroom.

- Select appropriate activities that include interaction with classmates. Use these activities to promote making friends and taking turns.

\section{Behavior}

- Regularly teach clear, simple rules that the child can achieve.

- When inappropriate behavior occurs, repeat the rule. Then encourage the student to engage in another activity.

- Praise the specific behavior whenever the person does something well, such as, Well done; now you know it.

- Ignore small disruptions and consistently praise appropriate behavior and improvement.

- Ask family members about unusual or inappropriate behavior. Parents can help you understand what the child is trying to communicate and how you can respond in a helpful way. 
- Do not expect too much too soon. Patience, consistency, and caring will eventually bring progress.

\section{Conclusion}

Inclusive Education will become a dynamic process if at least in any school there is a tendency to observe and follow the above-mentioned rules. It will move back and forth between theoretical discussion and practical implementation but finally it will get its destination and be of great help to those having SEN.

"The changes in the system of education are to mean that all learners should benefit and this will lead to the strengthening of civil society and the socio-economic well-being of all. Naturally this will guide the way to be more humane and enjoy the privileges of all equal societies around the world" (Clough, Corbett 2009:7).

\section{References:}

1. Clough, P.; Corbett, J. (2009) Theories of Inclusive Education, Institute of Education. University of London, UK: Sage.

2. Galstyan, M. (2013) An Assessment of Implementation of Inclusive Education in the Republic of Armenia. / Centre for Education Researches and Consulting, Yerevan. Available at: http://www.osf.am/wp-content/ uploads/2013/11/Inclusive-education-report [Accessed November 2018].

3. Stubbs, S. (2008) Inclusive Education: Where there are few recourses. / Ed. by I. Lewis, updated and revised version. Available at: <http://atlasalliansen.no/publication/inclusive-education-where-there-are-few-resour ces $>$ [Accessed December 2018].

4. Corps, H.; Ceralli, G.; Boisseau, S. (2012) Inclusive Education (Handicap International). Cambridge: CUP.

5. Alaverdyan, A.; Jean-Luc, S. (2009) Towards a More Equitable Society: A Guide to Inclusive Education Practices in Armenia, Yerevan.

6. (2011) World Report on Disability. / World Health Organization, Malta. 
7. Farrel, M (2000) Educational Inclusion. // British Journal of Special Education. N 27(1) pp. 35-38.

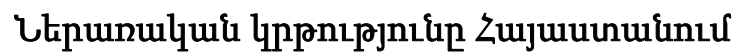

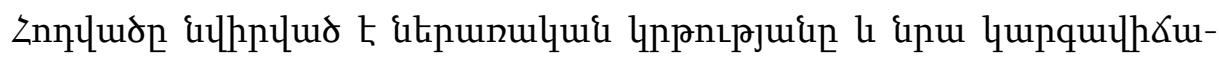

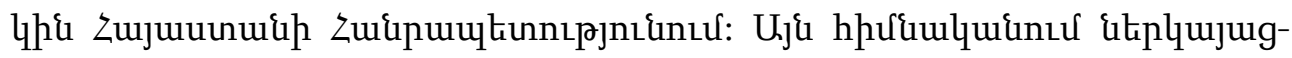

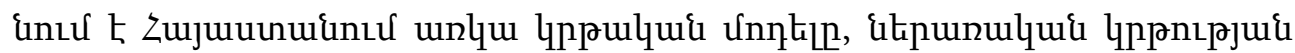

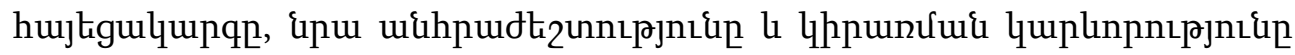

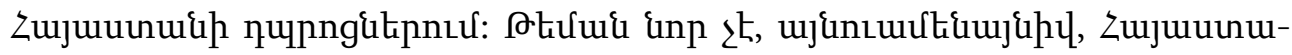

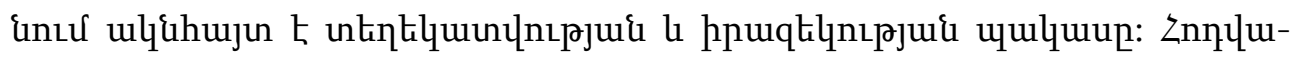

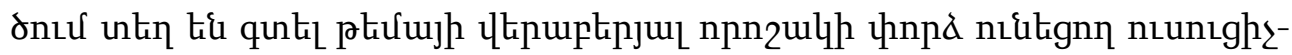

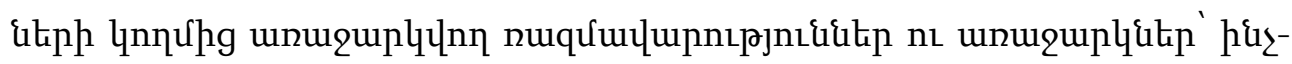

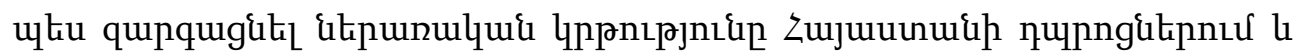

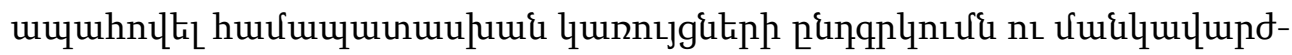
ateph lthuuumunpuuunnunp:

Received by the Editorial Board 22.01.2019

Recommended for publication by the reviewers 28.02.2019

Accepted for print 22.04.2019 